\title{
Contingent Existence and Iterated Modality
}

\author{
CIAN DORR
}

Boris Kment's Modality and Explanatory Reasoning is a rich and rewarding book. Along the way to his conclusions about the cognitive function of modal concepts and their relation to explanation, Kment presents many ideas which are of great independent interest. One example is in chapters 4 and 5, where Kment develops an admirably rigorous theory of worlds as classes of propositions (proper classes, in the case of metaphysically possible worlds). The most distinctive features of this theory stem from contingentism, the view that some things could have failed to exist, in the sense that there could have been nothing identical to them. According to Kment, propositions about contingent existents are themselves contingent, and thus worlds, as classes of propositions, end up being contingent as well.

So far Kment agrees with many other authors, including Adams (1981) and Stalnaker (2012). But there is one important point on which Kment agrees with Adams and disagrees with most other authors who have considered these questions. According to him, one upshot of all this contingency is that we have reject certain widely-accepted principles of propositional modal logic involving iterated modal operators. Abbreviating 'it is metaphysically necessary that $\varphi$ ' as ' $\square \varphi$ ’ and 'it is metaphysically possible that $\varphi$ ' as ' $\diamond \varphi$ ', the schemas Adams and Kment reject include the following:

(1) $\varphi \rightarrow \square \diamond \varphi$

(2) $\square(\square \sim \varphi \leftrightarrow \sim \diamond \varphi)$

(3) $\square \square \sim(\varphi \wedge \sim \varphi)$

(4) $\square(\varphi \rightarrow \diamond \varphi)$

(5) $\square \varphi \rightarrow \square \square \varphi$ 
On their view, given that Obama is a contingent existent, (1-3) are false if we substitute 'Obama is president' for $\varphi$; (1-4) are false if we substitute 'It is not the case that Obama is president'; and all five are false if we substitute 'Either Obama is president or it is not the case that Obama is president'. Even though these claims are not particularly crucial to Kment's larger program, they are quite striking and have broad ramifications for the current debate about contingentism (Williamson 2013). The main goal of this paper is to identify and evaluate Adams's and Kment's arguments against schemas like (1-5). At the end I will briefly argue against their view.

The immediate source of Adams's and Kment's rejection of (1-5) is their acceptance of what I will call
Adams's Schema
(a) $\square \forall x \square(\square \varphi(x) \rightarrow x$ exists $)$
(b) $\square \forall x \square(\diamond \varphi(x) \rightarrow x$ exists $)$

Adams would accept every sentence that results from (a) or (b) by replacing ' $\varphi(x)$ ' with any sentence in which the variable $x$ occurs free, and I believe Kment would too. But to motivate rejecting (1-5) we only need instances where $\varphi(x)$ is an atomic sentence involving $x$, or a truth-functional compound of such sentences. For example, the instance of (b) where ' $\varphi(x)$ ' is ' $x$ is president' implies that necessarily, if it is possible that Obama is president, then Obama exists. Given that it is not necessary that Obama exists, we can conclude from this that it is not necessarily possible that Obama is president, so that we have a counterexample to (1). ${ }^{1}$ Counterexamples to (2-4) can be extracted by similar reasoning from other simple instances of Adams's Schema.

But why are we supposed to accept Adams's Schema? One argument which one might be tempted to attribute to Kment turns on the idea that propositions cannot have properties, including modal properties, without existing. Abbreviating 'the proposition that $\varphi$ ' as ' $[\varphi]$ ', we can state the key premises of this argument as follows:

Mentioning Schema: For all $x,[\varphi(x)]$ mentions $x$ (at least where $\varphi(x)$ is a truth-functional compound of atomic sentences involving $x$ ).

\footnotetext{
${ }^{1}$ This reasoning turns on the K schema $\square(\varphi \rightarrow \psi) \rightarrow(\square \varphi \rightarrow \square \psi)$, which is not threatened by the Adams-Kment view.
} 
Dependence: If a proposition $p$ mentions an entity $x, \square$ (if $p$ exists, $x$ exists).

Instantiation Requires Existence (IRE): For any entity $x$ and property $f,[x$ has $f]$ logically entails [ $x$ exists] (Kment 2014: 78).

Atomic Operators: if $p=[\varphi]$, then $[\square \varphi]=[p$ has necessity $]$ and $[\diamond \varphi]=[p$ has possibility $]$.

Necessity Schema: $\square \varphi \leftrightarrow[\varphi]$ is necessary. ${ }^{2}$

Some explanation is required. Kent identifies propositions with "Lagadonian sentences": certain sets (or proper classes), thought of as sentences in a language where everything can serve as a name of itself, and every property or relation can also serve as a predicate expressing itself. The entities "mentioned" by a proposition are the entities that occur in it as names or predicates. Since all entities mentioned by a proposition belong to its transitive closure, Dependence falls out of the thesis that a class cannot exist unless each of its members exists. ${ }^{3}$

It is because propositions have this sentence-like structure that it makes sense to speak, as IRE does, of "logical entailment" among propositions. Like familiar relations of logical consequence among sentences of formal languages, the question whether $p$ logically entails $q$ depends only on the "logical forms" of $p$ and $q$, not on which particular entities they mention. We can express this idea more precisely by introducing a notion of substitution for propositions. When $f$ is a function such that $f(r)$ is an $n$-ary relation whenever $r$ is an $n$-ary relation, it is integral to Kment's theory of propositions that for any proposition $p$, there is a unique proposition call it the " $f$-image" of $p$ - that results from $p$ by replacing every occurrence in $p$ of an entity $x$ in the domain of $f$ (playing the role of a name for itself or non-logical predicate expressing itself)

\footnotetext{
${ }^{2}$ One could derive the Necessity Schema from Atomic Operators together with the Truth Schema, ' $[\varphi]$ is true $\leftrightarrow \varphi$ '. When ' $\mathrm{F}$ ' is a simple adjective expressing a property, Kment identifies [ $p$ is $\mathrm{F}]$ with $[p$ has $F$-ness] - so in particular, $[p$ is necessary $]=[p$ has necessity $]$. Atomic Operators thus implies that when $q=[\varphi],[q$ is necessary $]=[\square \varphi]$. Hence $[q$ is necessary] is true iff $[\square \varphi]$ is true, which implies by the Truth Schema that $q$ is necessary iff $\square \varphi$. However, the reasons for Kment to accept the Necessity Schema, and the analogous Possibility Schema ( $\diamond \varphi \leftrightarrow[\varphi]$ is possible'), look much stronger than the reasons to accept Atomic Operators.

${ }^{3}$ In fact the situation is slightly more complicated: Kment uses a coding trick to allow that proper classes can be mentioned despite not being members of anything. However, the proper classes mentioned by a proposition are all subclasses of its transitive closure, so we can still derive Dependence from the premise that a class cannot exist without any of its members or subclasses.
} 
with a corresponding occurrence of $f(x)$. The "formal" character of logical entailment can then expressed as follows: whenever $p p$ logically entail $q$, the $f$-images of $p p$ logically entail the $f$-image of $q$ for any bijection $f$. Many cases of necessitation thus fail to be cases of logical entailment: for example, if $f$ is the conjunction of two properties $g$ and $h$ distinct from itself, $[a$ has $f \rightarrow$ $a$ has $g$ ] will be necessary, although $[a$ has $f]$ does not logically entail $[a$ has $g]$. But according to Kment, every case of logical entailment is a case of necessitation: if $p$ logically entails $q$, the material conditional whose antecedent is $p$ and whose consequent is $q$ is necessary. Given this thesis, IRE and Atomic Operators jointly imply that when $p=[\varphi(x)]$, the material conditionals $[\square \varphi(x) \rightarrow p$ exists] and $[\diamond \varphi(x) \rightarrow p$ exists] are both necessary. So by the Necessity Schema, $\square(\square \varphi(x) \rightarrow p$ exists $)$ and $\square(\diamond \varphi(x) \rightarrow p$ exists $)$. But from the Mentioning Schema and Dependence, we know that $\square(p$ exists $\rightarrow x$ exists). So we can conclude that $\square(\square \varphi(x) \rightarrow x$ exists $)$ and $\square(\diamond \varphi(x) \rightarrow x$ exists $)$, using uncontroversial modal reasoning.

However, on reflection I doubt this argument captures Kment's actual motivation for Adams's Schema, since given his fine-grained theory of propositions, it would be quite surprising if he accepted Atomic Operators. To get a feel for the fine-grainedness of the view, consider that Kment would take all three of the following to be distinct:

$v_{1}:[a$ is a female fox $]$

$v_{2}:[a$ has $f]$, where $f=$ the property of being a female fox

$v_{3}$ : $a$ has the property of being a female fox]

Only $v_{2}$ is a subject-predicate proposition of the kind to which IRE applies. ${ }^{4} v_{1}$, unlike $v_{2}$, does not mention the property of being a female fox (a.k.a. the property of being a vixen). $v_{3}$ is something different again. On Kment's view, when 'is F' is syntactically complex, 'the property of being $F$ ' functions like the definite description 'the unique property to which it is essential that all and only the F things have it' (Kment 2013: 154). $v_{3}$ will thus come apart from $v_{2}$ at logically

\footnotetext{
${ }^{4} \mathrm{Kment}$ would identify $v_{2}$ with [ $a$ is a vixen].
} 
possible worlds at which some other property uniquely satisfies this description; it will come apart from $v_{1}$ at logically possible worlds at which no property satisfies the description.

Making these fine distinctions in the case of 'the property of being F' suggests that similar distinctions are called for in the case of 'the proposition that $\varphi$ '. For example, the considerations that lead Kment to distinguish $v_{2}$ from $v_{3}$ also seem to require distinguishing $o_{1}$ from $o_{2}$ :

$o_{1}: \quad[p$ is possible], where $p=[$ Obama is president $]$.

$o_{2}: \quad[[$ Obama is president $]$ is possible $]$.

$o_{1}$ is a subject-predicate proposition that mentions [Obama is president] and possibility, but not Obama. By analogy with $v_{3}$, I think Kment should say that $o_{2}$ is a different proposition, which does not mention [Obama is president], but rather picks it out descriptively in terms of some relations it bears to Obama and being president. For example, if Kment identified [Obama is president] with the ordered pair < being president, Obama>, he might identify $o_{2}$ with [the ordered pair whose first element is being president and whose second element is Obama is possible].

But if $o_{1}$ and $o_{2}$ are distinct, there is no obvious reason to follow Atomic Operators in identifying $o_{1}$ with $[\diamond($ Obama is president $)]-$ call this proposition $o^{*}$. Indeed, $o_{2}$ looks like a better candidate to be $o^{*}$, since this would allow Kment to accept the Mentioning Schema in full generality, whereas Atomic Operators requires restricting it to $\varphi(x)$ not involving modal operators.

While this blocks the argument for Adams's Schema based on IRE, identifying $o^{*}$ with $o_{2}$ would open up the possibility of a different argument for Adams's Schema based on a different principle closely related to IRE. Suppose for example that Kment went along with the suggestion that $o^{*}=o_{2}=$ [the ordered pair whose first element is being president and whose second element is Obama is possible], and furthermore adopted a Russellian theory of descriptions on which this proposition is in turn identical to $[\exists x(\operatorname{OrderedPair}(x) \wedge$ FirstElement $($ being president, $x) \wedge \operatorname{SecondElement(Obama,} x) \wedge \operatorname{Possible}(x) \wedge \forall \mathrm{y}((\operatorname{OrderedPair}(\mathrm{x}) \wedge$ FirstElement $($ being president, $x) \wedge$ SecondElement $($ Obama, $x)) \supset \mathrm{y}=\mathrm{x})]($ where the predications here are all atomic). Given that Kment regards free logic as a good guide to the logical entail- 
ment relation among propositions, this proposition logically entails $[\exists x($ SecondElement $($ Obama, $x))]$. And although IRE says nothing about this proposition (since it is not atomic), the motivation for IRE plausibly extends to existential quantifications of atomic propositions:

$\mathrm{IRE}^{+}:$For any entity $x$ and $n$-ary relation $r,\left[\exists y_{1} \ldots \exists y_{\mathrm{n}-1}\left(r\right.\right.$ applies to $\left.\left.y_{1}, \ldots, y_{n-1}, x\right)\right]$ logically entails $[x$ exists]. (Cf. principle (C4) in Adams 1981).

Thus, given assumptions in the spirit of his project, Kment could argue for the relevant instance of Adams's Schema from the premise that $o^{*}=o_{2}$. And there are other candidates to be $o^{*}$ which would serve equally well for this purpose. For example, an argument from IRE ${ }^{+}$could still be run if one identified $o^{*}$ with $o_{3}$ rather than $o_{2}$ :

$o_{3:}$ [[Obama is president] is true at some world in the sphere around the actualized world that contains all and only those worlds that match the actualized world with respect to the metaphysical laws].

$o_{3}$ would be a natural candidate for Kment to consider, since he holds that possibility is being true at some world in the unique sphere around the actualized world that contains all and only those worlds that match the actualized world with respect to the metaphysical laws. $o_{3}$ thus stands to $o_{2}$ much as $v_{2}$ stands to $v_{1}$; if it is true, its truth grounds the truth of $o_{2}$.

Still, why should $o^{*}$ be identified with any of these candidates? Kment is certainly not committed to a general program of identifying all propositions expressed using sentential operators with propositions about the properties of and relations among propositions. Consider [it is not the case that Obama is president $]$ - call it $n$. Kment certainly does not think that $n=[p$ has falsehood], where $p=$ [Obama is president], or that $n=$ [[Obama is president $]$ has falsehood $]$. Both of those propositions necessitate [Obama exists], whereas $n$ does not: the material conditional with $n$ as antecedent and [Obama exists] as consequent is false at possible worlds where 
Obama is never born. If the negation operator gets to work like this, why shouldn't modal operators get the same privilege?

Against the background of a theory of propositions as fine-grained as Kment's, claims of the form $[\varphi]=[\psi]$ are hard to argue for when $\varphi$ and $\psi$ are not syntactically isomorphic; one will face an uphill battle if one's strategy for arguing for Adams's Schema depends such controversial identifications. However, Adams's original paper also suggests a different argument for some controversial instances of Adams's Schema which requires no assumptions of this sort. Its key premise, in Adams's formulation, is that

... what is true about me at a world in which I do not exist must be determined, in accordance with some logical criterion, by the proposition that I do not exist, together with other propositions, true at that world, which are not about me (Adams 1981, 23).

This corresponds the following key assumption of Kment's system:

Supervenience*. For any propositions $p p$, if (i) for every $x, p p$ logically entail either [ $x$ exists] or [ $x$ doesn't exist] and (ii) every proposition that only mentions objects $x$ such that $p p$ logically entail $[x$ exists] is such that $p p$ either logically entail it or its negation, then $p p$ logically entail every proposition or its negation. (Kment 2013, 135).

Loosely following Adams $(1981,32)$, let's see how one can argue from Supervenience* to the following controversial consequence of Adams's Schema:

(*) $(\diamond$ (Obama is president $) \supset$ Obama exists $)$.

Choose some object $a$ such that necessarily, it is not possible for $a$ to be president. (Given standard essentialist assumptions there should be many such objects: Adams uses the premiere of Beethoven's 9th symphony.) By the Necessity Schema, $[\sim \diamond(a$ is president $)]$ is necessary, and so true at every possible world. ${ }^{5}$ We will argue that $[\sim \diamond($ Obama is president $)]$ is also true at pos-

\footnotetext{
${ }^{5}$ As Adams and Kment use 'true at', $p$ can be true at $w$ even when [ $p$ exists] is false at $w$. On Kment's analysis, $p$ is true at $w$ iff $p$ is logically entailed by the members of $w$ together with all propositions specifying the membership of proper classes.
} 
sible worlds where neither $a$ nor Obama exist. Let $w$ be such a world; let $p p$ be the propositions true at $w$ that don't mention Obama or $a$, together with [Obama doesn't exist] and [ $a$ doesn't exist]. Since every proposition is such that either it or its negation is true at $w$, Supervenience im- $^{-}$ plies that every proposition or its negation is logically entailed by $p p$, and hence that every proposition true at $w$ is logically entailed by $p p$. Since $[\sim \diamond(a$ is president $)]$ is true at $w$, it is logically entailed by $p p$. But recall that logical entailment is a formal relation, preserved by all substitution operations that do not collapse distinct objects to the same object. Consider the permutation $g$ on $\{$ Obama, $a\}$ where $g($ Obama $)=a$ and $g(a)=$ Obama. The $g$-image of any proposition $p$ is the result of replacing any occurrences of Obama in $p$ with occurrences of $a$, and vice versa. By the formality of logical entailment, the $g$-images of $p p$ logically entail the $g$-image of $[\sim \diamond(a$ is president)]. But since propositions that mention neither Obama nor $a$ are their own $g$-images, and [Obama doesn't exist] and [ $a$ doesn't exist] are one anothers' $g$-images, the $g$-images of $p p$ are just $p p$. It also seems plausible that the $g$-image of $[\sim \diamond(a$ is president $)])$ is $[\sim \diamond($ Obama is president $)] .{ }^{6}$ If so, $[\sim \diamond($ Obama is president $)]$ is logically entailed by $p p$, and so true at $w$. Thus $[\diamond($ Obama is president $)]$ is not true at any world where neither [ $a$ exists] nor [Obama exists] is true. This means that $[\diamond($ Obama is president $) \rightarrow$ Obama exists or $a$ exists $]$ is necessary; so by the Necessity Schema,

$\square(\diamond$ (Obama is president $) \rightarrow$ Obama exists or $a$ exists $)$

But given the arbitrariness in the choice of $a$, accepting this while rejecting $(*)$ is not on the cards. Indeed, by taking $a$ to be something like Obama's unit set, which couldn't exist without Obama, we can complete the argument for $(*)$.

It is easy to turn this into an argument for $\square \forall x \square(\diamond(x$ is president $) \rightarrow x$ exists $)$, and parallel arguments can be given for many other instances of Adams's Schema where $\varphi(x)$ is intuitively

\footnotetext{
${ }^{6}$ This conflicts with Atomic Operators, according to which $[\sim \diamond($ Obama is president $)]$ is the negation of an atomic proposition which mentions neither Obama nor $a$, and is thus its own $g$-image. To make room for Atomic Operators, one could instead use $g^{+}$where $g^{+}(\mathrm{Obama})=a, g^{+}(a)=$ Obama, $g^{+}([$Obama is president $])=[a$ is president $]$ and $g^{+}([a$ is president $]$ ) $=[$ Obama is president $]$.
} 
"positive". However the strategy does not work for "negative" instances like " $\square \forall x \square(\diamond \sim(x$ is president $) \rightarrow x$ exists)', since it would require finding an object $a$ such that $\square \sim \diamond \sim(a$ is president), and there is no reason to believe in such a thing. Because of this limitation, the argument poses no obvious threat to schemas like (2-5). But (*) is still controversial enough, since it forces contingentists to reject the B-schema (1).

This argument has two major weaknesses. One is its reliance on Supervenience*, which is a very strong claim because logical entailment is such a strong relation. Since many intuitively watertight arguments - e.g. from [There are bachelors] to [There are unmarried men], or even from [ $p$ is necessary] to [ $p$ is possible] - fail to be logical entailments, we lack a strong pre-theoretical grip on the contrast between logical entailment and metaphysical necessitation. So even if we grant that something like Supervenience* is true, it is reasonable to demand an argument that the truth in the vicinity is Supervenience* itself rather than the analogous principle involving metaphysical necessitation in place of logical entailment. I didn't find anything in Kment's explanation of his reasons for Supervenience* (Kment 2013,82) that looked like it could play this discriminating role.

The second weakness is the assumption that $[\sim \diamond($ Obama is president $)]$ is the $g$-image of $[\sim \diamond(a$ is president $)]$, or - equivalently, since that proposition does not mention Obama - the result of substituting Obama for $a$ in it. What general principle could justify this? We might be tempted by the following:

Strong Substitution Schema: $[\varphi(b)]$ is the result of substituting $b$ for $a$ in $[\varphi(a)]$.

But Kment certainly cannot accept this. For him, [Hesperus is brighter than Phosphorus] is identical to [Venus is brighter than Venus]; substituting Mars for Hesperus in it yields [Mars is brighter than Mars], not [Mars is brighter than Phosphorus]. Fortunately, there is an easy restriction that avoids this problem:

Restricted Substitution Schema: If for some $x$, $[\varphi(x)]$ does not mention $a$, then $[\varphi(b)]$ is the result of substituting $b$ for $a$ in $[\varphi(a)]$. 
This gets around the counterexample, since there is no $x$ for which $[x$ is brighter than Phosphorus] fails to mention Hesperus. And since there certainly are objects $x$ for which $[\diamond(x$ is president)] fails to mention $a$, the Restricted Substitution Schema is still strong enough to support the claim that $[\sim \diamond($ Obama is president $)]$ is the result of substituting Obama for $a$ in $[\sim \diamond(a$ is president)].

However, the Restricted Substitution Schema is deeply problematic, since it leads to inconsistency in conjunction with assumptions at least as plausible as itself. When $x$ is a name or variable, let ' $\mathrm{R}(x)$ ' abbreviate ' $x$ is a proposition that mentions some proposition $q$ such that the result of substituting $x$ for $q$ in $x$ is not true'. ${ }^{7}$ Let $s$ be [snow is white]; let $t$ be $[\mathrm{R}(s)]$. ( $t$ is false, since $s$ doesn't mention any propositions.) Since the expression abbreviated by ' $\mathrm{R}(x)$ ' contains no names or free variables other than $x$, it seems obvious that there is no proposition mentioned by $[\mathrm{R}(x)]$ for all $x$. So we can invoke the Restricted Substitution Schema to deduce that for any propositions $p$ and $q$, $[\mathrm{R}(q)]$ is the result of substituting $q$ for $p$ in $[\mathrm{R}(p)]$. In particular, $[\mathrm{R}(t)]$ is the result of substituting $t$ for $s$ in $t$. But the result of substituting one object for another in any proposition mentions everything that proposition mentions, except for the substituted object. It follows that $s$ is the one and only proposition mentioned by $t$, since any other proposition mentioned by $t$ would have to be mentioned by $[\mathrm{R}(x)]$ for all $x$. So if $[\mathrm{R}(t)]$ is true, no proposition $q$ mentioned by $t$ is such that the result of substituting $t$ for $q$ in $t$ is not true: in other words, it is not the case that $\mathrm{R}(t)$. Meanwhile, if $[\mathrm{R}(t)]$ is not true, then there $i s$ a proposition mentioned by $t-$ namely $s$-such that the result of substituting $t$ for it in $t$ is not true: in other words, $\mathrm{R}(t)$. So we have established the following disjunction:

Either $[\mathrm{R}(t)]$ is true and not $\mathrm{R}(t)$, or $[\mathrm{R}(t)]$ is not true and $\mathrm{R}(t)$.

And this is inconsistent with the Truth Schema ' $[\varphi]$ is true if and only if $\varphi$ ', a principle which seems absolutely basic to our thinking about propositions. ${ }^{8}$

\footnotetext{
${ }^{7}$ Since ' $\mathrm{R}(x)$ ' is a mere abbreviation, it is irrelevant whether there is a property of being an $x$ such that $\mathrm{R}(x)$.

${ }^{8}$ Kment rejects the necessitation of the Truth Schema, but that is a different matter.
} 
Of course, the Truth Schema is not beyond all doubt - one might reject it in response to the semantic paradoxes, though in my view a better response is to reject disquotation schemas like “" $\varphi$ ” expresses $[\varphi]$ and nothing else'. But it is hard to see why anyone who rejected the Truth Schema would accept the Necessity Schema, which also played a crucial role in the argument from Supervenience* to $(*)$. So that argument is on shaky ground no matter what. ${ }^{9}$

The foregoing argument - essentially a version of the Russell-Myhill paradox (see Goodman forthcoming) - shows that 'structured' conceptions of propositions like Kment's are more problematic than has generally been acknowledged. While it does not refute the thesis that propositions are Lagadonian sentences, it undermines many of its applications, by forcing us to reject natural generalisations relating the structure of the proposition $[\varphi]$ to that of the English sentence ' $\varphi$ '. Somehow, $[\mathrm{R}(t)]$ is not the result of substituting $t$ for $s$ in $[\mathrm{R}(s)]$. If we can get used to this, why not also deny that $[\diamond(a$ is president $)]$ is the result of substituting $a$ for Obama in $[\diamond($ Obama is president $)] ?^{10}$

I conclude that contingentists have not yet been given a compelling reason to reject schemas like (1-5). Nevertheless, the Adams-Kment view is clearly worth taking seriously, and is in some ways attractive - there is certainly something disquieting about the way in which the combination of contingentism, essentialism and the B-schema requires, as it were, non-existent objects to be able to differ modally without any underlying categorical differences. And I don't want to suggest that any of (1-5) are so obviously correct that their failure is a very serious problem in its

\footnotetext{
${ }^{9}$ It is even more obvious that the Necessity Schema stands or falls with the Possibility Schema (see note 2). And the foregoing argument against the Restricted Substitution Schema can be modified to rely on the Possibility Schema rather than the Truth Schema. Let ' $\mathrm{R}^{*}(x)$ ' abbreviate ' $x$ mentions some proposition $q$ such that the result of substituting $x$ for $q$ in $x$ is not possible'. By the same reasoning as before, the Restricted Substitution Schema yields that either $\left[\mathrm{R}^{*}(t)\right]$ is possible and not $\mathrm{R}^{*}(t)$, or $\left[\mathrm{R}^{*}(t)\right]$ is not possible and $\mathrm{R}^{*}(t)$. But we can also argue that $\mathrm{R}^{*}(t)$ iff $\diamond \mathrm{R}^{*}(t)$, from the following premise (which Kment would accept): for any $p, q, r, s$, if $\diamond(p$ is possible) then $p$ is possible, and if $\diamond(p$ mentions $q$ and nothing else) then $p$ mentions $q$ and nothing else, and if $\diamond(q$ is the result of substituting $r$ for $s$ in $t$ ) then $q$ is the result of substituting $r$ for $s$ in $t$. We thus arrive at a disjunction ruled out by the Possibility Schema: either $\left[\mathrm{R}^{*}(t)\right]$ is possible and not $\diamond \mathrm{R}^{*}(t)$ or $\left[\mathrm{R}^{*}(t)\right]$ is not possible and $\diamond \mathrm{R}^{*}(t)$.

${ }^{10}$ There is a different route to the conclusion that the structures of propositions do not match the structures of the sentences that express them, involving plural quantification. Surprisingly, given that he is so careful to allow for singular propositions about proper classes, Kment lacks a theory of "singular propositions about pluralities". Given standard plural logic, there must be cases where $[\varphi(x x)]=[\varphi(y y)]$ even though at least one of $x x$ is not one of $y y$, for any formula $\varphi(x x)$ with a free plural variable.
} 
own right. Claims involving iterated modal operators are notoriously hard to express idiomatically in natural languages, so it seems perfectly appropriate for our assessment of them to be driven by systematic considerations. However, I think that there is a serious problem in the vicinity, since the considerations that motivate rejecting (1-5) also seem to require rejecting certain ordinary judgments involving iterated counterfactuals. Consider:

(6) If Obama had never been born, some unfortunate people who would have voted for Obama if he had been on the ballot in 2008 would never have taken part in politics.

This is perfectly ordinary English, and is plausibly true. ${ }^{11}$ But the motivations for rejecting (1-5) which we have considered seem to carry over to (6). Anyone who accepted Atomic Operators would have just as much reason to identify [If it were that $\varphi$ then it would be that $\psi$ ] with [p bears $r$ to $q]$, where $p=[\varphi], q=[\psi]$ and $r$ is the relation of counterfactual dependence. Similarly, if one identified $[\square \varphi]$ and $[\diamond \varphi]$ with propositions picking out $[\varphi]$ descriptively in terms of its constituents, one would have equal reason to identify [If it were that $\varphi$ then it would be that $\psi$ ] with some proposition picking out $[\varphi]$ and $[\psi]$ descriptively in terms of their constituents. And finally, the substitution-theoretic argument for $\left(^{*}\right)$ can easily be turned into an argument against (6). Consider something that would not have existed if Obama had never been born - say, Obama's daughter Malia. Whatever reason there might be to think that $[\sim \diamond$ (Obama is president)] is the result of substituting Obama for $a$ in $[\sim \diamond(a$ is president $)]$, there is equally good reason to think that [Some unfortunate people who would have voted for Malia if she had been on the ballot in 2008 never take part in the political process] is the result of substituting Malia for Obama in [Some unfortunate people who would have voted for Obama if he had been on the ballot in 2008 never take part in the political process]. But Supervenience* implies that propositions that interchange two objects always agree at worlds where neither object exists, so that if (6) is true, (7) is also true:

\footnotetext{
11 The reading of (6) I have in mind is the one where the quantifier takes scope under the conditional, so that it is consistent with there not being any unfortunate people.
} 
(7) If Obama had never been born, some unfortunate people who would have voted for Malia if she had been on the ballot in 2008 would never have taken part in the political process.

But this is bad news: Malia was only ten years old in 2008, so (7) is much less likely to be true than (6) is. ${ }^{12}$

Adams's and Kment's combination of contingentism with Adams's Schema is thus far more deeply at odds with ordinary thought then they recognize. If there were a compelling argument for Adams's Schema, this would be bad news for contingentism. But as we have seen, the extant arguments are quite problematic. So for now, the biggest challenges for contingentists lie elsewhere.

New York University

New York 11215, U.S.A.

cian.dorr@nyu.edu

\section{References}

Adams, R. M. 1981. Actualism and thisness. Synthese 49: 3-41.

Goodman, J. Forthcoming. Reality is not structured. Analysis.

Kment, B. 2014. Modality and Explanatory Reasoning. Oxford: OUP.

Stalnaker, R. 2012. Mere Possibilities: Metaphysical Foundations of Modal Semantics. Princeton: Princeton University Press.

Williamson, T, 2013. Modal Logic as Metaphysics. Oxford: OUP.

\footnotetext{
12 The problem is perhaps even sharper if we consider sentences in which 'actually' appears under a conditional, like 'If Obama had never been born, some unfortunate people who actually voted for Obama in 2008 would never have taken part in politics'. The salient reading of this is true if, for example, John and Mary voted for Obama in 2008, and if Obama had never been born, one or other of them would have been unfortunate and not taken part in politics. Explaining this within Kment's system already looks hard; doing so without undermining the arguments against (15) looks impossible.
} 